\title{
Übersicht
}

\author{
Birgit Vogel-Heuser*, Jay Lee, and Paulo Leitão
}

\section{Agents enabling cyber-physical production systems}

\section{Softwareagenten zur Realisierung von Cyber-physischen Produktionssystemen}

DOI 10.1515/auto-2014-1153

Received October 6, 2014; accepted December 19, 2014

\begin{abstract}
In order to be prepared for future challenges facing the industrial production domain, Cyber-Physical Production Systems (CPPS) consisting of intelligent entities which collaborate and exchange information globally are being proclaimed recently as part of Industrie 4.0. In this article the requirements of CPPS and abilities of agents as enabling technology are discussed. The applicability of agents for realizing CPPS is exemplarily shown based on three selected use cases with different requirements regarding real-time and dependability. The paper finally concludes with opportunities and open research issues that need to be faced in order to achieve agent-based CPPSs.
\end{abstract}

Keywords: Cyber-physical production systems, Industrie 4.0, agent, multi-agent systems.

Zusammenfassung: Cyber-Physical Production Systems (CPPS) sind intelligente, kooperierende Produktionseinheiten, welche Informationen global austauschen. Die Anwendbarkeit der Agententechnologie zur Realisierung von CPPS'en wird an Hand von drei internationalen Fallstudien für Teile von CPPS gezeigt, um die Vorteile und die $\mathrm{zu}$ bearbeitenden Forschungsfragen auch in Bezug auf Echtzeit- und Zuverlässigkeitsanforderungen zu identifizieren.

Schlüsselwörter: Cyber-physische Produktionssysteme, Industrie 4.0, Softwareagent, Multi-Agent-Systeme.

\footnotetext{
*Corresponding author: Birgit Vogel-Heuser, Technische Universität München, Institute of Automation and Information Systems, 85748 Garching near Munich, e-mail:vogel-heuser@ais.mw.tum.de Jay Lee: NSF I/UCRC on Intelligent Maintenance Systems (IMS), Univ. of Cincinnati, Cincinnati

Paulo Leitão: Polytechnic Institute of Bragança, Bragança
}

\section{Introduction}

Various roadmaps from Europe and the United States concerning research and developments towards future factory automation [1-3] and Digital Manufacturing and Design Innovation [4] conclude that both information and communication will be key issues for future production systems. Roadmaps envision production systems being able to flexibly adjust their behavior to address changing conditions [5], e.g. business conditions such as changing demand or product portfolio, or technical conditions, e.g. technical faults, and provide novel sophisticated mechanisms, which are enabled by available communication and realized by significantly increased intelligence of computing entities. To apply Cyber-Physical Systems within the production automation domain, various application scenarios have been identified, e.g. production networks [6], maintenance and diagnosis [7, 8], or dynamic reconfiguration $[9,10]$. These use cases arise from an exhaustive use of information exchange, coordination and collaboration between Cyber-Physical entities. The application of state of the art information technology in combination with traditional, established engineering processes for vertical and horizontal integration of information, but enlarged by cross facility and company cooperation e.g. along the supply chain is today referred to as Industrie 4.0 in Germany.

Research regarding agent technology was initially conducted within the field of artificial intelligence some decades ago [11, 12]. Agents are typically characterized as communicating, collaborative, intelligent entities applied for distributed problem solving [10]. The application of agent technology within various fields of production automation, e.g. distributed production planning and scheduling as well as process supervision, is being investigated and implemented for many years now [13-16]. In contrast, applying agents for dependable, real-time automation software directly on field level [43] considering hard real-time requirements is a comparatively novel re- 
search field in production automation $[16,18,19]$. Agents hence became an adequate means to provide desired flexibility while coping with complex problems efficiently. In addition, agent technology was first introduced for performance monitoring in computer-integrated manufacturing [20].

Accordingly, the efforts towards realizing Industrie 4.0 enabled by Cyber-Physical Systems might be facilitated by adopting agent technology. Therefore, in the subsequent Section 2, characteristics of Cyber-Physical Systems in the production automation domain, i.e. Cyber-Physical Production Systems (CPPS) [6, 21] and agent technology are introduced. Some examples of agent technology being successfully applied to typical Industrie 4.0 use cases in the production automation domain are presented in Section 3. These application scenarios are used to identify opportunities and open research issues that need to be solved in order to realize CPPS by applying agent technologies.

\section{Agents enabling CPPS: state of the art}

Within this section, CPPS and their key characteristics enabling Industrie 4.0 are introduced briefly at first. In this paper, CPPS are considered to be equivalent with Industrie 4.0 in the domain of production automation. In the remainder, agents in the domain of production automation are defined and some existing agent technologies, architectures and their key characteristics as well as the challenges and opportunities applying agents in CPPS are discussed. Finally, the similarity between the intensively studied agent technologies and the vision of CPPSs is juxtaposed.

\subsection{Cyber-physical production systems}

Industry 4.0 is based on Cyber-Physical Production Systems (CPPS) which can be based on a $5 \mathrm{C}$ architecture (connection, conversion, cyber, cognition, and configuration) [22] (Figure 1).

In the "Connection" level, devices can be designed to self-connect and self-sensing for its behavior. In the "Conversion" level, data from self-connected devices and sensors are measuring the features of critical issues with selfaware capabilities, machines can use the self-aware information to self-predict their potential issues. In the "Cyber" level, each machine is creating its own "twin" by using these instrumented features and further characterize the machine health pattern based on a "Time-Machine" agent. The established "twin" in the cyber space can perform self-compare for peer-to-peer performance for further synthesis. In the "Cognition" level, the outcomes of selfassessment and self-evaluation will be presented to users based on an "infographic" meaning to show the content and context of the potential issues. In the "Configuration" level, the machine or production system can be reconfigured based on the priority and risk criteria to achieve resilient performance [23, 24].

Embedded systems are able to monitor and control physical processes by sensors and actuators. CPS are Embedded Systems, but are networked with each other to utilize globally or locally in another CPS available information sources and services [6]. Accordingly, CPSs combine the vision of intelligent, adaptive control systems with seamless vertical, horizontal and dynamic information exchange between heterogeneous plattforms [6].

The traditional way of engineering and operating production systems is based on static information flows within and in between automation software, from automation software to manufacturing operations management. When introducing CPPS, it will be necessary to establish information exchange between heterogeneous components - e.g. field devices, automation devices, MES but also pure information systems. An intensively discussed and applied approach for facilitating information exchange between a priori unknown, heterogeneous components and information sources is the use of terminologies [25] and semantic technologies [5]. Therein, data can be enhanced with semantics using respective ontologies to make contents understandable for both humans and machines. Unfortunately, although quite common in the classical information technology domain, ontologies are only partially built up and therefore not established in the production automation domain $[10,26]$ despite a strict terminology in the field of Industrie 4.0 is inevitable [27].

Requirement $\mathbf{R}_{\mathbf{C P P S} 1}$ : In order to apply CPPSs sucessfully, knowledge regarding their structure e.g. resources like machines with their characteristics, their interfaces to other facilities inside a CPPS, and operations they provide for the manufacturing of products and operation are necessary.

In order to react to changing (external and internal) conditions like unforeseen failures during operation or varying customer demand, CPPSs have to be able to adjust their structure and their behavior [6]. Here, the traditional way of implementing automation software based on assumptions about structure and static behavior of production facilities cannot address this requirement suficciently any more [10]. 


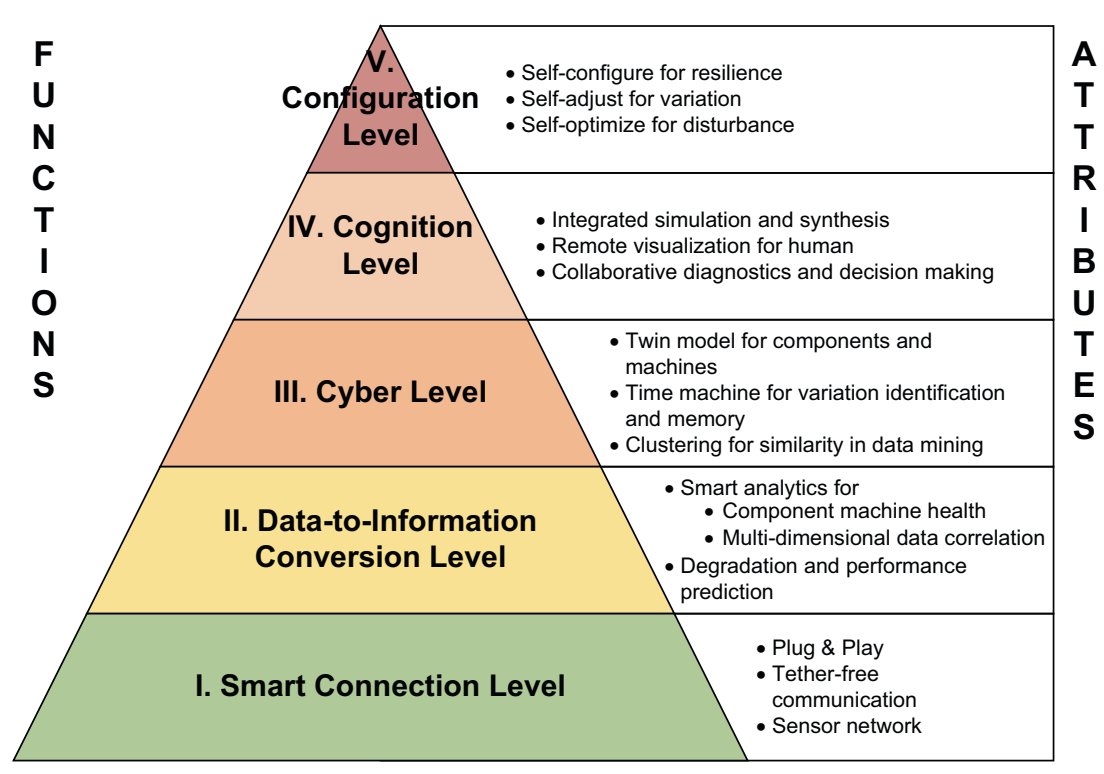

Figure 1: 5 C Architecture of CPPS [21].

Requirement $\mathbf{R}_{\text {CPPS2 }}$ : CPPS are able to intelligently behave and flexibly adapt their behavior and structure in order to react to before runtime unknown changing (external and internal) conditions .

CPPSs have to be adjustable to various (external) conditions like the manufacturing of novel products. Here, adjusting solely the behavior will not be sufficient. Therefore, the structure of a CPPS will not be static along its lifecylce and has to be adjusted as required to react best to changing conditions. CPPS may be composed to higher level CPPS. In some works also the term cyber-physical component is used [28]. Nevertheless the structure may be also totally flat, i.e. non-hierarchical.

Requirements $\mathbf{R}_{\text {CPPS3 }}$ : The structure of CPPSs needs to be adaptable. Thereby, no assumption about nonhierarchical and/or hierarchical structures of production facilities should be made.

To be sure, these challenges are only an exemplary selection of various different requirements of CPPS. The selection focus on their necessity for argumentation in the remainder of this article. A detailed, exhaustive discussion of the various CPPS characteristics is provided for example in $[6,21,29]$.

Despite the fact that the levels of the former automation pyramid are no longer valid, there are still different requirements regarding dependability and real-time to be fulfilled for field and control tasks on the one hand and MES including recipe scheduling for new orders on the other hand. To achieve dependability and real-time specific automation platforms e.g. PLCs are implemented with cyclic scheduling to meet also the maintainabiliy on a level of technicians.
Requirements $\mathbf{R}_{\text {CPPS4 }}$ : Automation for CPPSs needs to fulfill real-time and dependability requriments on specific platforms to be maintainable for maintenance personel.

\subsection{Multi-agent systems and agent technology}

Multi-agent systems (MAS) [30, 31] is a computational paradigm introduced in the distributed artificial intelligence field, characterized by the decentralization and parallel execution of activities based on autonomous agents. MAS solutions replace the centralized control by a distributed functioning where the interactions among agents lead to the emergence of "intelligent" global behavior, being able to react and adapt to condition changes without external intervention [30]. The decentralization of control functions over distributed autonomous and cooperative agents facilitates modularity, autonomy, flexibility, robustness and adaptability.

Ability A A $_{\text {MS1 }}$ : Multiple agents can form a distributed, decentralized system referred to as multi-agent system. The basic class of MAS is non-hierarchical, but in different applications also hierarchical MAS are used e.g. a coordination agent to realize decisions in real-time [32] or a supervision agent (see myJoghurt application [6]).

An agent can be defined as an "autonomous component that represents physical or logical objects in the system, capable to act in order to achieve its goals, and being able to interact with other agents, when it does not possess knowledge and skills to reach alone its objectives" [13]. Moreover, an agent can sense its environment and make 
decisions according to its internal behavior, knowledge and objectives. Aiming to address the emergent challenges of self-organization and responsiveness, besides the basic properties, i.e. autonomy, intelligence and cooperation, an agent is required to provide a set of self-X properties ( $\mathrm{X}$ is a placeholder for "one or more desirable properties of a system subjected to a variable operation condition" [33]). The application of self-X properties in production automation has been intensively discussed over the last years $[34,35]$. A prerequisite to realize self-X properties is the agents' self-awareness.

Ability $\mathbf{A}_{\text {MAS2}}$ : An agent is characterized by intelligent behavior and self-awareness by applying knowledge about its skills and the environment (depending on the agent's architecture).

Agents can be understood as self-aware, intelligent building blocks ( $\mathbf{A}_{\text {MAS2 }}$ ). Similarly, CPPS are demanded to be flexibly adaptable and intelligent ( $\left.\mathbf{R}_{\mathbf{C P P S} 2}\right)$. Agents realize their intelligent behavior by means of internal knowledge about their skills and capabilities ( $\mathbf{A}_{\text {MAS2 }}$ ). Thus, key requirements for CPPS, namely $\mathbf{R}_{\text {CPPS1 }}$ and $\mathbf{R}_{\text {CPPS2 }}$ can be realized by means of agents due to their inherent abilities $\left(\mathbf{A}_{\text {MAS2 }}\right)$. Inproduction automation, a system consists not solely of software but also of a physical part consisting of e.g. mechanical and electrical aspects.

Multi-agent systems are often applied for distributed production planning [36]. For example, the approach presented in [37] combines production planning with a flexible control of production resources by means of agents. Therein, a resource agent controls a production resource deductively. An agent in context of production automation can be seen as a defined entity which is intended to reach its objectives independently but cooperatively while interacting with its environment including other agents [19, 38].

The aggregation of the informational part (i.e. the agent) and the physical hardware part (e.g. a robot, a machine) in production automation may be defined as a holon. A holon, as Koestler devised the term, is an identifiable part of a (manufacturing) system that has a unique identity, yet is made up of sub-ordinate parts and in turn is part of a larger whole [39].

Typically holons consist of an informational and a physical part and on higher levels a holon defines recursively a set of holons which perfectly matches with the requirement that automation production comprises software and hardware components.

The essence of the holonic approach is the capability to decompose a complex problem into stable intermediate sub-problems, using hierarchy structures. PROSA [40] and ADACOR [41] are two examples of architectures that explore the holonic principles.

Ability $\mathrm{A}_{\text {MAS3 }}$ : The holonic paradigm facilitates the recursive structuring: a holon can be a self-contained whole to its subordinated parts and simultaneously a dependent part when seen from higher levels (i.e. a single agent as well as a whole MAS).

As identified in $\mathbf{R}_{\text {CPPS3 }}$, CPPS are required to be flexibly structurable without assumption about an underlying logical structure. Agents are characterized as a distributed system $\left(\mathbf{A}_{\text {MAS1 }}\right)$ with heterarchical structures $\left(\mathbf{A}_{\text {MAS1 }}\right)$. Therefore, agents provide sufficient means to realize the desired CPPS requirement $\mathbf{R}_{\text {CPPS3 }}$.

In $[42,43]$ a multi-agent system following this definition is presented which enables dependable production in presence of sensor failures. Agents are dedicated to physical equipment, e.g. machine parts, to be controlled [43]. Agents exchange their knowledge about related sensor data in real-time to calculate virtual sensors in case of sensor degradation or faults, to increase machine availability by operating with the virtual sensor with lower precision instead. Thus automation agents are closely related to the physical layer of a plant.

To fulfill real-time and dependability requirements as well as maintainability ( $\left.\mathbf{R}_{\mathbf{C P P S}}\right)$, additional measures need to be taken, e.g. assure communication between agents which fulfills real-time requirements and avoid agents' actions with unknown results. The agent paradigm with negotiations in between agents as well as learning and acting on the agent's knowledge is restricted to safe actions [17, 42]. The implementation on Programmable Logic Controllers (PLCs) is also discussed in Section 3.

Ability $\mathbf{A}_{\text {MAS4 }}$ : Agents representing physical devices and their functions at the field level need to fulfill real-time and dependability requirements by limiting their actions to the action space. They are implemented on specific platforms to achieve maintainability and real-time behavior in ms.

In a nutshell, all requirements of CPPSs described in detail in Section 2.1 can be realized by existing agent technologies as presented in Section 2.2 due to their inherent characteristics.

Besides agents, the service-oriented architecture is explicitely proposed in the context of the efforts towards Industrie 4.0 [44] as well as CPPS [45]. In the production automation domain, first investigations of the serviceoriented paradigm already exist [46]. The service-oriented architecture and agent technologies can be seen as complementary approaches with different possible ways to combine them [47]. An exemplary combination of both, 
the service-oriented paradigm and agent technology will be presented in Section 3.3.1.

\section{Applying agent technology for realizing cyber-physical production systems}

As identified in the previous section, the inherent characteristics of agent technologies can provide sufficient means to realize CPPS. A variety of agent approaches in industrial automation already exist (see [14, 16, 48] for surveys). According to the survey of Leitão et al. [16] "There are areas where agents have been successfully deployed and other areas where they have not broken through. The former one is the production planning, scheduling, and logistics where agents bring measurable benefits in terms of better resource utilization, shorter delivery times, fuel savings, etc." [...] "The latter one is the factory automation, where agent-based systems are still deployed only in laboratorial environments or as industrial prototypes. There are still barriers, either technology- or human factor-related, that obstruct the adoption of these novel paradigms at large scale. Interestingly, both the industrial agents community and the community around serviceoriented automation, have identified identical issues. The major technological roadblock is the inability of the new technology to respect contemporary industrial requirements for real-time capabilities, robustness, availability of mature engineering tools, safety, and standardization." Leitão et al. [16] also mentioned the convergence of agentbased and service-oriented architectures as a future trend as well as agent learning.

Within this section, three applications of agent technology are described in detail in context of CPPS to demon- strate this hypothesis in a practical manner. The first and the second scenario belong to the area of production optimization and quality control, whereas the third scenario targets also agent learning and real-time reconfiguration on industrial PLCs using IEC 61131-3 supported by an engineering approach [49].

\subsection{The Watchdog Agent@: intelligent data analytics for self-aware machine and predictive maintenance}

For providing more transparency to manufacturing assets' capabilities and finally improve e.g. up time and production quality, predictive health management arises due to novel options provided by CPPS like global data exchange [50, 51]. In this context, the Watchdog Agent ${ }^{\circledR}$ was developed [52], which consists of different analytics methodologies to assess degradation process of machine and components, e.g. sensors. An overview of the Watchdog Agent ${ }^{\circledR}$ concept is depicted in Figure 2. It is able to predict machine health degradation with self-aware intelligence, and therefore prevent potential issues or failure.

Towards achieving its goal, the Watchdog Agent ${ }^{\circledR}$ is collecting and integrating a huge amount of data of various production facilities and different platforms [53]. For predictive analytics, a toolbox of well-known methodologies for processing, prediction and forecasting [52] was implemented (cp. Figure 2). Based on its knowledge about the process and pieces of equipment to be monitored, the agent is aware of the current situation which facilitates to adjust available tools for predictive analytics dynamically. Furthermore, the agent is able to memorize observed situations and, by that, is able to identify situations that were never observed before. Thus, the Watchdog Agent ${ }^{\circledR}$

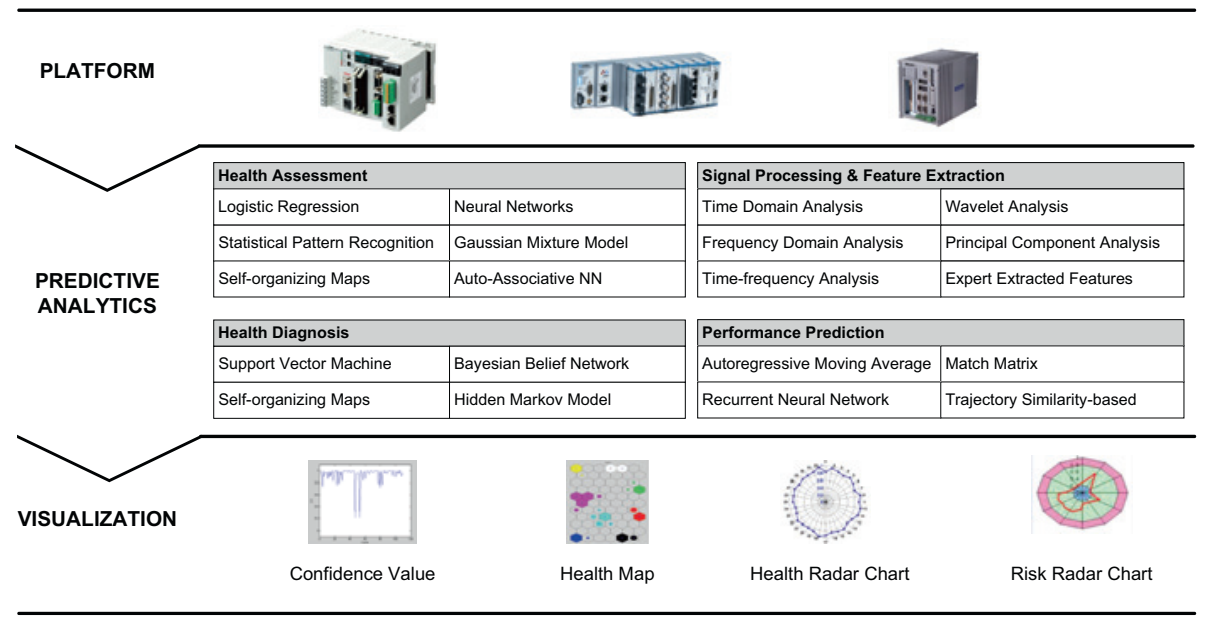

Figure 2: Internal Architecture of the Watchdog Agent ${ }^{\circledR}$ with available tools for predictive analytics and visualization. 
exhibits typical elements of agent characteristics for intelligent, self-aware behavior (cp. $\mathbf{A}_{\text {MAS2 }}$ ). The different kind of knowledge models used by analytics tools are applied to realize an intelligent, predictive behavior $\left(\mathbf{R}_{\mathbf{C P P S} 2}\right)$ of the Watchdog Agent ${ }^{\circledR}$. Thus, the Watchdog Agent ${ }^{\circledR}$ is realizing a typical CPPS scenario by means of agent techniques. Real-time requirements depend on the application of the watchdog agents ( $\mathbf{R}_{\mathbf{C P P S}}$ ), but the algorithm are not applied on the field control level.

\subsection{Multi-agent system integrating process and quality control}

Ensuring constant quality of products is a very important but often time-consuming effort in industrial automation. In case of a high variety of products the complexity of quality control increases. Therefore, the main objective of the EU FP7 GRACE project (InteGration of pRocess and quAlity Control using multi-agEnt technology) [54] was to implement a flexible, distributed system which integrates process monitoring and quality control. In order to achieve this goal, a modular, intelligent, collaborative and distributed control system was developed, using the MAS principles and operating in an industrial factory plant producing laundry washing machines. The installed solution contributes for the maximization of the factory profitability by applying self-adaptation procedures at local and global levels to face unexpected condition changes.
Having this in mind, an ecosystem of autonomous agents representing the manufacturing components disposed along a production line was considered, grouped in the following types of agents according to their similarities (see [55] for more details): Product Type Agent (PTA), Product Agents (PA), Machine Agents (MA) and Independent Meta Agents (IMA). PTAs represent the catalogue of products that can be produced by the production line, i.e. the washing machine models, possessing the knowledge related to the product model and process plan. Product agents handle the production of product instances along the production line, each PA managing the on-line production of one product washing machine. Machine agents represent the physical resources disposed along the production line, such as robots, quality control stations and operators. IMAs implement global supervisory control to such distributed structure, e.g. optimizing and adapting global policies for the system.

The global system objectives emerge from the cooperation among individual agents, each one contributing with its local behavior, as illustrated in Figure 3. In such distributed systems, the use of ontologies is crucial as already mentioned in 2.1 to establish a common understanding among the agents by defining the vocabulary and the semantics of the shared knowledge. This conforms to the typical characteristics $\mathbf{A}_{\text {MAS1 }}$ and $\mathbf{A}_{\text {MAS2 }}$ of agents as well as the desired requirements of CPPS $\mathbf{R}_{\mathbf{C P P S} 1}$. For this purpose, an ontology was designed and implemented considering the particularities of the home appliance domain and the integration of process and quality control levels.

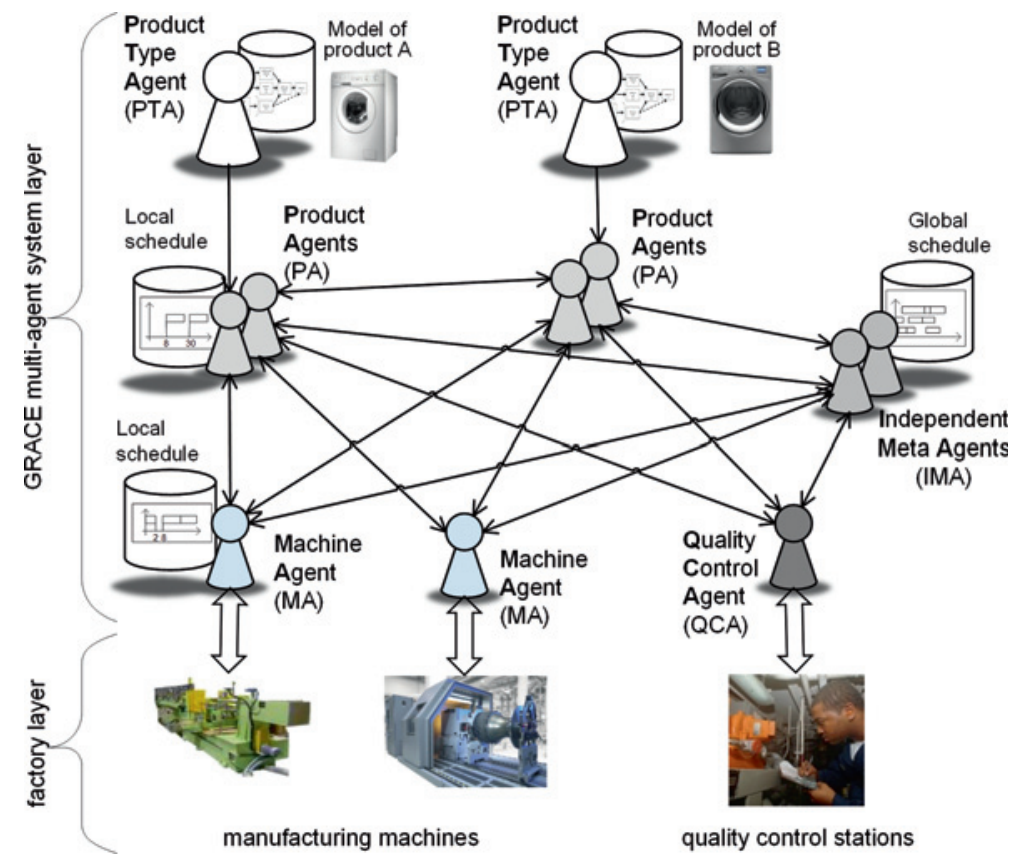

Figure 3: GRACE multi-agent system architecture according to [55]. 
The interaction patterns aiming at the integration of process and quality control for the implementation of selfadaptation procedures based on feedback control loops are of special relevance in this work. Examples of such designed procedures are the dynamic adaptation of the functional test plan, the customization of the on-board controller parameters, the analysis of the evolution of the quality indexes at a specific station and the on-line adaptation of the process plans for each washing machine model. Accordingly, characteristics of self-aware and adaptable behavior of agents (ability $\mathbf{A}_{\text {MAS2 }}$ ) realize the CPPS requirement $\mathbf{R}_{\text {CPPS2 }}$ ).

An example of application of the self-adaptation procedures is the customization of the functional test plan, which is performed at the Functional Test Area located near the end of the production line and managed by the Quality Control Agent (QCA). Currently, this operation lasts 6 minutes and comprises a fixed plan, even if some tests may be redundant according to the results gathered from previous inspection tests. PA agents are continuously collecting information about the execution of process operations (e.g. bearing insertion or welding operations) and testing operations (e.g. control gap or assembly visual check) by interacting with MA agents associated to these process or quality control stations. When the product arrives to the functional test area, the PA agent correlates the gathered information to adapt the sequence of the tests defined in the plan by removing unnecessary tests, adjusting others or customizing the messages provided to the operators. The implementation of this self-adaptation mechanism allows reducing the inspection time by approximately $20 \%$ and also improving the product quality by executing more efficient and accurate inspection tests.

The GRACE multi-agent system infrastructure was implemented by using the JADE (Java Agent DEvelopment Framework) framework [56]. The deployed multi-agent system infrastructure was intensively tested in the factory plant and the achieved results showed an increase of the production and energy efficiency, an increase of the product quality, as well as a reduction of the production downtimes, the scrap costs and non-conformities. The adaptation is performed at local and global levels, with different real-time granularity levels. In fact, the adaptation performed by RA and PA agents are mainly related to soft realtime but IMA agents are performing self-optimization procedures in background without real-time costraints.

\subsection{Dynamic reconfiguration for flexible, self-healing production}

For evaluating novel approaches in the field of CPPS, the demonstrator myJoghurt was developed [6] which consists of several loosely coupled production plants forming a CPPS network. All participants within the CPPS network are represented by agents: for instance, customer agents are responsible for the representation of a customer's demands and plant agents represent single plants. The demonstrator is named after its exemplary, primary good to be produced: mass customized yoghurt. Various demonstration scenarios can be exhibited in myJoghurt: Two of them will be used as application example in the remainder of this subsection.

\subsubsection{A multi-agent system for mass customized production}

In a first application, mass customized yoghurt production is considered. After an order has been created by a customer, the order is passed to the CPPS network. Subsequently, the CPPS network needs to identify the resource being involved and subsequently needs to plan the production process. Within an open CPPS network, companies typically do not want to share all information about production capacity and capability. Therefore, a distributed coordination approach that enables an automated planning of the production process which is currently under development.

Independent of the concrete coordination mechanisms, an information model is needed which enables an agent identifying whether certain process steps can be realized by its associated plant or plant component. Within the myJoghurt demonstrator, for instance plant agents represent the individual CPPS and must be able to identify, whether their functionalities are sufficient to provide a certain topping configuration and whether a specific required process step, e.g. bottling, can be fulfilled by the associated physical component.

In the field of service-oriented manufacturing systems [46], this challenge, namely matching services required by a customer's order with services and possible service orchestrations that hence fulfill a technical process to be realized, is referred to as Manufacturing Service Matching Problem (MSMP) [57]. To perform the MSMP using existing approaches, models that describe all technical processes realized by a plant have to be defined.

In [58] and [6], a knowledge-based approach benefits from a major assumption: Instead of describing all possi- 


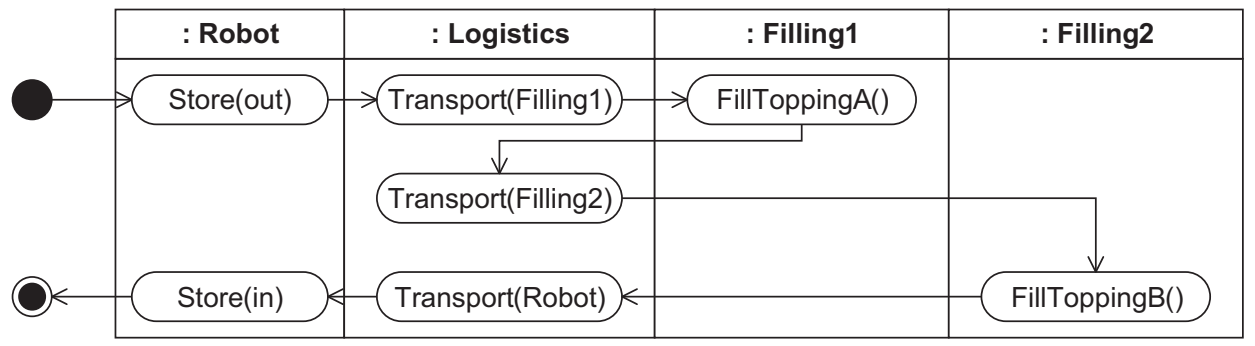

Figure 4: Exemplary operation strategy for yoghurt with topping $A$ and topping $B$. ble technical processes to be realized, it is distinguished between "what the CPPS is required to do" - i.e. the product to be produced and the technical process to be realized (customer's order provided by the customer agent) and "what the CPPS is able to do" - i.e. the functionalities a physical system provides (services of a plant agent). Using the provided functionalities, the overall state space of the CPPS can be computed automatically and, by that, it can be derived whether a certain technical process can be realized. Moreover, an adequate control software's configuration that is able to perform the desired process, namely an operation strategy, can be generated (cf. Figure 4).

The operation strategy is passed to the supervision agent which in turn configures the execution agents responsible to execute the plant agents' services, cp. Figure 5. Hence, such execution agents can be seen as orchestration engines that compose field-level automation services provided by plant agents to produce a certain product as negotiated within the CPPS network.

As exemplary depicted in Figure 5 for the logistics agent, these agents might be in turn a set of agents in a holonic way (e.g. a set of conveyor agents and switch agents realize the logistics agent). The approach can therefore be used within the myJoghurt CPPS application to determine an operation strategy that enables the production of yoghurt according to an arbitrary order initiated by a customer. As an alternative structure in agent@PLC by Wannagat [43], Schütz [49] and Ulewciz [59] (Figure 6) the CPPS plant agent is composed of a system agent knowing the actual plants structure, the communication agent, responsible for messaging between CPPS, a process agent containing the production process and necessary substeps, the whiteboard with the jobs and its' states to be fulfilled as well as the automation control agent, representing the physical part of the plant and scheduling the necessary sub-steps for the process agent.

In Summary, a multi agent system is applied here to successfully realize a typical CPPS scenario with flexible intelligent behavior adoption due to external conditions (customer request) according to CPPS key requirement $\mathbf{R}_{\text {CPPS2 }}$. The realization is based upon knowledge about a physical plant's operations (cp. agents' ability $\mathbf{A}_{\text {MAS1}}$ ).

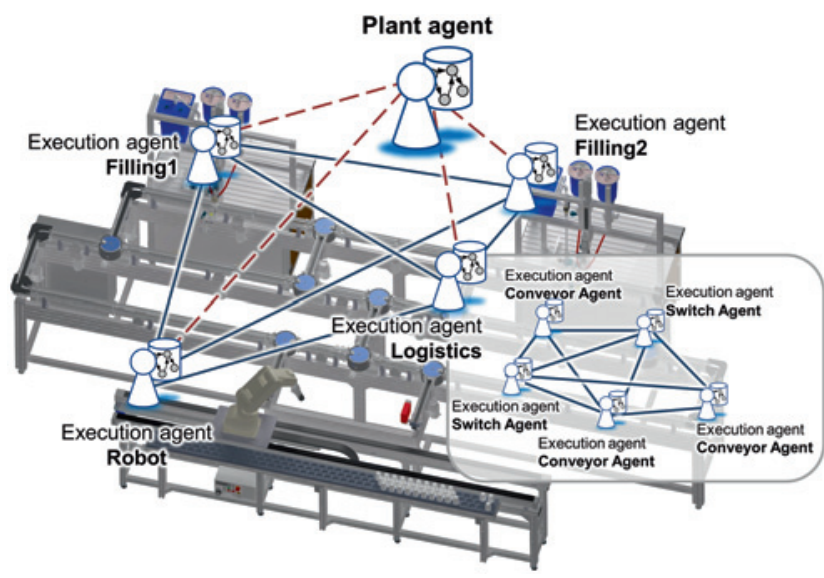

Figure 5: Overview on the proposed multi-agent system architecture for the myJoghurt CPPS (solid lines indicate real-time communication; dashed lines non-real-time communication).

The flexible adaptation of the automation systems behavior to produce a mass customized good is achieved by means of a (holonic) multi agent system according to the characteristics of $\mathbf{A}_{\text {MAS3 }}$. Even if real-time is not an issue in most of the cases in this use case, the agent on PLC was implemented with a cycle time of $4 \mathrm{~ms}$ for cap engraving (CoDeSys Soft PLC as well as Beckhoff CX) ${ }^{1}$ and fulfills

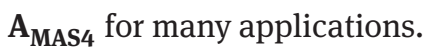

\subsubsection{Agent-based dynamic alarm management}

When operating a plant, typically more notifications, i.e. warnings, and alarms, are generated by production facilities than can be physically perceived and addressed by human operators. These floods of notifications result from causally related notifications (i.e. dependent notification sequences) which are triggered mostly by a single disturbance. This hinders human personal to quickly perceive critical situations, i.e. situations resulting in eco-

$1 \mathrm{http}: / /$ de.codesys.com/das-system.html; http://www.beckhoff.de/ default.asp?twincat/twincat-3.htm 


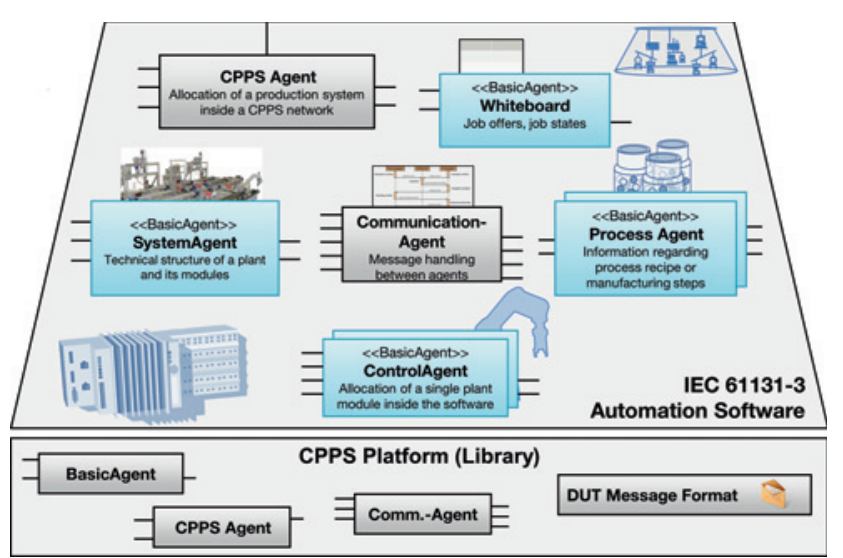

Figure 6: Structure of the IEC 61131-3 agent@PLC.

nomic, material or personal damage, which are indicated by sequences of notifications. To make things worse, more than $50 \%$ of identified notification sequences are caused by misconfigurations of the alarm management system [8], because dependencies between notifications are difficult to be recognized by the application engineer during engineering. Flexible production systems leverage this challenge to foresee operational dependencies correctly during engineering. For example, when producing yoghurt within the myJoghurt demonstrator, analysis identified that notification sequences depend on the different cream stage to be produced because the varying viscosity of the yoghurt change timing parameters within the process engineering part. Accordingly, in CPPS systems, where recipes can be flexibly changed during operation, a dynamic, intelligent alarm management system is required. To tackle this challenge, an agent-based approach extending the traditional alarm management system's functionality was investigated in order to reduce the floods of notifications significantly (concept see Figure 7).

The alarm management agent filters and aggregates notifications. Based on a finite automaton [60] forming the agent's knowledge base, the discrete notification streams of the operating plant are analyzed; filters and classifiers are used to further reduce number of relevant notification sequences. Finally, solely critical situations, i.e. critical notification sequences, are visualized to the operator which facilitates him to react to alarms quickly by reducing the number of notifications and consequently the management effort significantly. Here, the critical component is the agent's knowledge base: the agent has to visualize all critical situations dependably while filtering and aggregating the notifications restrictively. Therefore, a human in the loop machine learning approach was chosen which enables to automatically detect notification sequences while being supervised by humans. Historical alarm logs are used for identifying significant notification sequences based on statistical pattern recognition techniques to suppress redundant notifications and visualize the cirital situations [61]. Typically, machine learning approaches are challenged by statistically non-perfect learning data. In order to avoid misconfigurations of the alarm agent e.g. by too restrictive notification sequences, automatically identified notification sequences are verified/falsified by interacting with the plant's operator. As identified in [8], applying machine learning to historical alarm data would result in various notification sequences

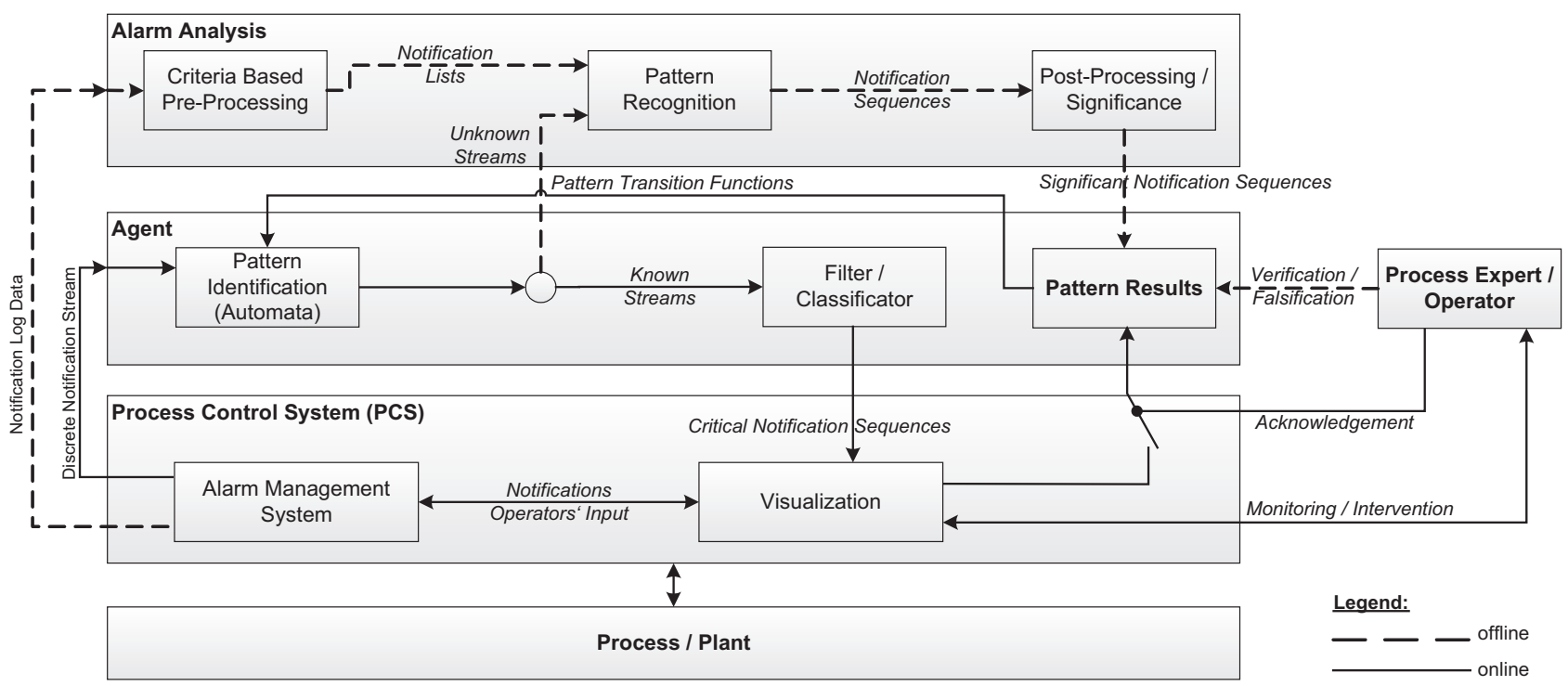

Figure 7: Overview of the building blocks for intelligent, dynamic alarm management using agent oriented pattern identification. 
which are statistically correct but logically nonsense. In order to reduce the operators' effort, criteria based preprocessing grounded on background knowledge like reference designation and plant documentation is applied. Additionally, post-processing based on significance tests is applied to further reduce the number of patterns to be revised by the operator. When identifying unknown sequences of notifications during operation, the pattern recognition is applied online to dynamically extend the knowledge base. This enables handling dynamically varying notification data based on different recipes to be produced as typical within the CPPS myJoghurt demonstrator.

In a nutshell, applying a knowledge base (cp. requirement $\mathbf{R}_{\mathbf{C P P S} 2}$ and $\mathbf{A}_{\text {MAS2 }}$ ) to realize intelligent behavior and learning, facilitates dynamically adjusting the alarm management in real-time during runtime and finally increases the dependability of CPPS. The real-time capabilities of the algorithm implemented in Matlab/Simulink on a PC-based PLC are still being evaluated.

\section{Challenges towards a multi-agent architecture for cyber-physical production systems}

In this article, a discussion of CPPSs' requirements and inherent abilities of agent technologies were provided. Whereas CPPSs are a concept of intelligent, globally connected and information exchanging production systems, agents can be seen as the enabling technology due to their inherent abilities which are required for realizing CPPSs. Research on agent technologies have been conducted for years. Various applications of agent technologies for nonreal-time applications exist like predictive maintenance (cp. the exemplary application described in Section 3.1) or machine supervision (cp. Section 3.2). In contrast, for applying agent technology in hard real-time environments to increase availability promising approaches exist (e.g. the applications presented within Section 3.3).

Besides rising standards for connection of the plant level to the Cloud as OPC/UA interfaces (Object Linking and Embedding (OLE) for Process Control/Unified Architecture) for future Industrie 4.0 systems also migration concepts for existing plants are inevitable due to the long operation of plant between 20 and 50 years [62]. Therefore converting entities are required to enable such existing plants to act in Industrie 4.0 compliant systems or CPPS networks like the myJoghurt demonstrator. Herein, agents can mediate between OPC/UA or others and classical automation structure and networks like Profibus and other fieldbus systems by acting as wrapper of legacy systems. For that matter, the agents provide the necessary and accessible information of the plant, providing a data exchange platform and access rights to data agents allow to bridge the gap until Industrie 4.0 standards are available and to adapt to changing standards.

In this contribution, the application of agents for providing intelligent behavior of CPPS during operation was focused. But additionally, CPPS with typical characteristics of intelligent behavior based on desired knowledge challenges the engineering of CPPS [28]. Firstly, intelligent behavior has to be considered during design. Secondly, required knowledge which can be used during operation has to be defined sufficiently during engineering. An exemplary application which combines aspects of engineering and operating by means of machine learning was introduced in Section 3.3, but there is still a lack in the knowledge representation for more complex systems executable during runtime as well as learning algorithm executable under real-time constraints both, PLC or PCbased field level automation devices. Also an integrated modeling approach for CPPS interacting with each other is missing.

Acknowledgement: We would like to grateful thank the members of the IEEE Technical Committee on Industrial Agents as well as the members of the VDE/VDI-GMA 5.15 Technical Committee on Multi-agent systems in industrial automation for valuable discussions.

\section{References}

1. European Commission: MANUFUTURE strategic research agenda - assuring the future of manufacturing in Europe. 2006.

2. European Factory of the Future Association: Factories of the future PPP FoF 2020 roadmap - Consultation Document. 2012.

3. Acatech: Cyper-physical Systems - driving force for innovation in mobility, health, energy and production - Position Paper. 2011.

4. Office of the Press Secretary: President Obama Announces Two New Public-Private Manufacturing Innovation Institutes and Launches the First of Four New Manufacturing Innovation Institute Competitions. URL: http://www.whitehouse.gov/thepress-office/2014/02/25/president-obama-announces-twonew-public-private-manufacturing-innovatio. [Accessed: 29Sep-2014].

5. C. Legat, S. Lamparter, and B. Vogel-Heuser: Knowledge-Based Technologies for Future Factory Engineering and Control. In: Service Orientation in Holonic and Multi Agent Manufacturing and Robotics, pp. 355-374, Springer Berlin Heidelberg, 2013.

6. B. Vogel-Heuser, C. Diedrich, D. Pantförder, and P. Göhner: Coupling heterogeneous production systems by a multi-agent 
based cyber-physical production system. In: IEEE International Conference on Industrial Informatics (INDIN), 2014.

7. J. Lee and $H$. Wang: New technologies for maintenance. In: Complex System Maintenance Handbook, pp. 49-78, Springer, London 2008.

8. J. Folmer and B. Vogel-Heuser: Computing dependent industrial alarms for alarm flood reduction. In: International MultiConference on Systems, Signals \& Devices 2012, pp. 1-6.

9. C. Legat and B. Vogel-Heuser: An Orchestration Engine for Services-oriented Field Level Automation Software. In: Workshop on Service Orientation in Holonic and Multi-Agent Manufacturing, 2014.

10. C. Legat, C. Seitz, S. Lamparter, and S. Feldmann: Semantics to the Shop Floor: Towards Ontology Modularization and Reuse in the Automation Domain. In: 19th IFAC World Congress 2014, pp. 3444-3449.

11. G. Weiss: Multiagent Systems: a modern approach to distributed artificial intelligence. MIT Press, 1999.

12. S. Russel and P. Norvig: Artificial Intelligence: A modern approach (Ed. 3), Peason, 2010.

13. P. Leitão: Agent-based distributed manufacturing control: A state-of-the-art survey. In: Eng. Appl. Artif. Intell. 22 (2009), No. 7, pp. 979-991.

14. M. Metzger and G. Polakow: A Survey on Applications of Agent Technology in Industrial Process Control. In: IEEE Trans. Ind. Informatics 7 (2011), No. 4, pp. 570-581.

15. W. Shen, Q. Hao, H. J. Yoon, and D. H. Norrie: Applications of agent-based systems in intelligent manufacturing: An updated review. In: Adv. Eng. Informatics 20 (2006), No. 4, pp. 415-431.

16. P. Leitão, V. Marik, and P. Vrba: Past, Present, and Future of Industrial Agent Applications. In: IEEE Trans. Ind. Informatics 9 (2013), No. 4, pp. 2360-2372.

17. Wannagat, A.: Entwicklung und Evaluation agentenorientierter Automatisierungssysteme zur Erhöhung der Flexibilität und Zuverlässigkeit von Produktionsanlagen. Ph.D. Thesis, Technische Universität München, 2010.

18. P. A. Urbano, T. Wagner, P. Gohner, U. Katzke, and B. VogelHeuser: Introducing reliability and real-time features in flexible agent-oriented automation systems. In: 2nd IEEE Int. Conf. Ind. Informatics (INDIN) 2004.

19. B. Vogel-Heuser, P. Göhner, and A. Lüder: Agent based control of production systems - and its architectural challenges. In: Industrial Agents: Emerging Applications of Software Agents in Industry, 2015.

20. J. Lee: Machine performance monitoring and proactive maintenance in computer-integrated manufacturing: review and perspective. In: Int. J. Comput. Integr. Manuf. 8 (1995), No. 5 , pp. 370-380.

21. B. Vogel-Heuser, C. Diedrich, and M. Broy: Anforderungen an CPS aus Sicht der Automatisierungstechnik. In: at Automatisierungstechnik 61 (2013), No. 10, pp. 669-676.

22. B. Bagheri: 5 C Architecture, Introduced by IMS Center for CyberPhysical Systems in Manufacturing. http://www.imscenter.net/ cyber-physical-platform [Accessed: 12-Dez-2014].

23. J. Lee, B. Bagheri, and H.-A. Kao: Recent Advances and Trends of Cyber-Physical Systems and Big Data Analytics in Industrial Informatics. In: IEEE Int. Conference on Industrial Informatics (INDIN), 2014.

24. J. Lee, E. Lapira, B. Bagheri, and H.-A. Kao: Recent advances and trends in predictive manufacturing systems in big data environment. In Manufacturing Letters 1 (2013), No. 1, pp. 3841.

25. A. Yurdakil, E. Schneider, and M. Hodon: Standardisation of international and interdisciplinary terminology in the language of transportation and automation engineering. In: 21st EUROZEL International Symposium, 2013.

26. M. Muehlhause, N. Suchold, and C. Diedrich: Application of semantic technologies in engineering processes for manufacturing systems. In: 10th IFAC Workshop on Intelligent Manufacturing Systems 2010, pp. 54-59.

27. J. Pfrommer, M. Schleipen, T. Usländer, U. Epple, R. Heidel, L. Urbas, $O$. Sauer, and J. Beyerer: Begrifflichkeiten um Industrie 4.0?: Ordnung im Sprachwirrwarr. In: Entwurf komplexer Automatisierungssysteme - EKA 2014?: Beschreibungsmittel, Methoden, Werkzeuge und Anwendungen?; 13. Fachtagung mit Tutorium, Eds.: U. Jumar, C. Diedrich, p. 8, Magdeburg, 2014.

28. S. Feldmann, S. Rösch, D. Schütz, and B. Vogel-Heuser: ModelDriven Engineering and Semantic Technologies for the Design of Cyber-Physical Systems. In: IFAC Workshop on Intelligent Manufacturing Systems 2013, pp. 210-215.

29. M. Riedl, M. Meier, H. Zipper, and C. Diedrich: Automation Meets CPS. In: IFAC Workshop on Intelligent Manufacturing Systems (IMS) 2013, pp. 216-221.

30. M. Wooldridge: An Introduction to Multi-Agent Systems. John Wiley \& Sons, 2002.

31. J. Ferber, Multi-Agent Systems: An Introduction to Distributed Artificial Intelligence. Addison Wesley, 1999.

32. Vogel-Heuser, B., Diedrich, C., Fay, A., Jeschke, S., Kowalewski, S., Wollschläger, M., and Göhner, P.: Challenges for Software Engineering in Automation. In: Journal of Software Engineering and Applications 7 (2014), No. 5.

33. M. Onori, D. Semere, and B. Lindberg: Evolvable systems: an approach to self-X production. In: International Journal of Computer Integrated Manufacturing 24 (2011), no. 5. pp. 506-516.

34. P. Leitão: Self-Organization in Manufacturing Systems: Challenges and Opportunities. In: IEEE International Conference on Self-Adaptive and Self-Organizing Systems Workshops 2008, pp. 174-179.

35. S. Bousbia and D. Trentesaux: Self-organization in distributed manufacturing control: state-of-the-art and future trends. In: IEEE Int. Conf. Syst. Man Cybern. 5, 2002.

36. W. Shen, L. Wang, and Q. Hao: Agent-based distributed manufacturing process planning and scheduling: a state-of-the-art survey. In: IEEE Trans. Syst. Man Cybern. Part C. 36 (2006), No. 4, pp. 563-577.

37. A. Lüder, J. Peschke, T. Sauter, S. Deter, and D. Diep: Distributed intelligence for plant automation based on multi-agent systems: the PABADIS approach. In: Prod. Plan. Control 15 (2004), No. 2, pp. 201-212.

38. VDI/VDE, VDI/VDE: guideline 2653 - multi agent systems in industrial automation - part 1: Fundamentals. 2010.

39. A. Koestler: The Ghost in the Machine. Arkana Books, London 1969.

40. H. Van Brussel, J. Wyns, P. Valckenaers, L. Bongaerts, and P. Peeters: Reference architecture for holonic manufacturing systems: PROSA. In: Comput. Ind 37 (1998), No. 3, pp. 255274.

41. P. Leitão and F. Restivo: ADACOR: A holonic architecture for agile and adaptive manufacturing control. In: Comput. Ind. 57 (2006), No. 2, pp. 121-130. 
42. A. Wannagat and B. Vogel-Heuser: Agent oriented softwaredevelopment for networked embedded systems with real time and dependability requirements in the domain of automation. In: 17th IFAC World Congress, 2008.

43. D. Schutz, A. Wannagat, C. Legat, and B. Vogel-Heuser: Development of PLC-Based Software for Increasing the Dependability of Production Automation Systems. In: IEEE Trans. Ind. Informatics 9 (2013), No. 4, pp. 2397-2406.

44. H. Kagermann, W. Wahlster, and J. Helbig: Deutschlands Zukunft als Produktionsstandort sichern Umsetzungsempfehlungen für das Zukunftsprojekt Industrie 4.0 Abschlussbericht des Arbeitskreises Industrie 4.0. Berlin, 2013.

45. D. Zühlke and L. Ollinger: Agile Automation Systems Based on Cyber-Physical Systems and Service-Oriented Architectures. In: Advances in Automation and Robotics Vol. 1, Lecture Notes in Electrical Engineering 122, pp. 567-574, Ed.: G. Lee, Springer Berlin Heidelberg, 2012.

46. F. Jammes and H. Smit: Service-Oriented Paradigms in Industrial Automation. In: IEEE Trans. Ind. Informatics 1 (2005), No. 1, pp. 62-70.

47. J. M. Mendes, P. Leitão, F. Restivo, and A. W. Colombo: Serviceoriented agents for collaborative industrial automation and production systems. In: Lecture Notes in Computer Science (including subseries Lecture Notes in Artificial Intelligence and Lecture Notes in Bioinformatics), LNAI 5696 (2009), pp. 13-24.

48. R. W. Brennan: Toward Real-Time Distributed Intelligent Control: A Survey of Research Themes and Applications. In: IEEE Trans. Syst. Man Cybern. Part C (Applications Rev. 37 (2007), No. 5, pp. 744-765.

49. D. Schütz: Automatische Generierung von Softwareagenten für die industrielle Automatisierungstechnik der Steuerungsebene des Maschinen- und Anlagenbaus auf Basis der Systems Modeling Language. Ph.D. Thesis, Technische Universität München, 2014.

50. J. Lee: E-manufacturing-fundamental, tools, and transformation. In: Robot. Comput. Integr. Manuf. 19 (2003), No. 6, pp. 501-507.

51. J. Lee, B. Bagheri, and H.-A. Kao: Cyber-Integrated Big Data Analytics Agent for Industry 4.0 Application. In: 6. Expertenforum Agenten im Umfeld von Industrie 4.0 2014, pp. 99-107.

52. D. Djurdjanovic, J. Lee, and J. Ni: Watchdog Agent-an infotronics-based prognostics approach for product performance degradation assessment and prediction. In: Adv. Eng. Informatics 17 (2003), No. 3-4, pp. 109-125.

53. J. Lee, H.-A. Kao, and S. Yang: Service Innovation and Smart Analytics for Industry 4.0 and Big Data Environment. Procedia CIRP 16 (2014), pp. 3-8.

54. GRACE-InteGration of pRocess and quAlity Control using multiagEnt technology. http://grace-project.org/ [Accessed: 21-Aug2014].

55. N. Rodrigues, A. Pereira, and P. Leitão: Adaptive Multi-Agent System for a Washing Machine Production Line. In: 6th International Conference on Industrial Applications of Holonic and Multi-Agent Systems 2013, pp. 212-223.

56. F. Bellifemine, G. Caire, and D. Greenwood: Developing MultiAgent Systems with JADE. John Wiley \& Sons, 2007.

57. C. Legat, S. Lamparter, and C. Seitz: Service-oriented productdriven manufacturing. In: IFAC Intelligent Manufacturing Systems 10, 2011.
58. C. Legat, D. Schütz, and B. Vogel-Heuser: Automatic generation of field control strategies for supporting (re-)engineering of manufacturing systems. In: J. Intell. Manuf. 25 (2013), pp. 11011111.

59. S. Ulewicz, D. Schütz, and B. Vogel-Heuser: Integration of Distributed Hybrid Multi-Agent Systems into an Industrial IT Environment. In: 12th IEEE International Conference on Industrial Informatics (INDIN) 2014, pp. 519-525.

60. J. Folmer, U. Katzke, D. Pantfoerder, B.-M. Pfeiffer, and B. VogelHeuser: Method for Analyzing Message Archives and Corresponding Computer Program. PCT/EP2009/0035232010, 2012.

61. J. Folmer, F. Schuricht, and B. Vogel-Heuser: Detection of Temporal Dependencies in Alarm Time Series of Industrial Plants. In: 19th IFAC World Congress 2014, pp. 1-6.

62. B. Vogel-Heuser, J. Folmer, and C. Legat: Anforderungen an die Softwareevolution in der Automatisierung des Maschinen- und Anlagenbaus. In: at - Automatisierungstechnik 62 (2014), No. 3, pp. 163-174.

\section{Bionotes}

\section{Prof. Dr.-Ing. Birgit Vogel-Heuser}

Technische Universität München, Institute of Automation and Information Systems, Boltzmannstr. 15, 85748 Garching near Munich

vogel-heuser@ais.mw.tum.de

Prof. Dr.-Ing. Birgit Vogel-Heuser graduated in electrical engineering and received the Ph.D. in mechanical engineering from the RWTH Aachen in 1991. She worked for nearly ten years in industrial automation in the machine and plant manufacturing industry. After holding different chairs of automation she has been head of the Institute of Automation and Information Systems at the Technische Universität München since 2009. Her research work is focused on modeling and education in automation engineering for distributed and intelligent systems.

\section{Prof. Jay Lee, D.Sc}

NSF I/UCRC on Intelligent Maintenance Systems (IMS), Univ. of Cincinnati, Cincinnati, PH 45221

jay.lee@uc.edu

Prof. Jay Lee, D.Sc is Ohio Eminent Scholar, L.W. Scott Alter Chair Professor, and Distinguished University Research Professor at the University of Cincinnati and is founding director of National Science Foundation (NSF) Industry/University Cooperative Research Center (I/UCRC) on Intelligent Maintenance Systems which is a multicampus NSF Industry/University Cooperative Research Center. He is a Fellow of ASME, SME, as well as a founding fellow of International Society of Engineering Asset Management (ISEAM). He serves as editors and associate editor for a number of journals including IEEE Transaction on Industrial Informatics, Int. Journal on Prognostics \& Health Management (IJPHM), Int. Journal on Service Operations and Informatics. His current work focuses on Industrial Big Data Analytics and Smart Manufacturing Systems for Industrie 4.0 Transformation. 


\section{Prof. Paulo Leitão}

Polytechnic Institute of Bragança, Campus Sta Apolónia, Apartado 1134, P-5301-857 Bragança, Portugal

pleitao@ipb.pt

Prof. Paulo Leitão received the MSc and PhD degrees in Electrical and Computer Engineering, both from the University of Porto, in 1997 and 2004, respectively. He is Head of the Department of Electrical Engineering at the Polytechnic Institute of Bragança and member of the Artificial Intelligence and Computer Science Laboratory. He is Chair of the IEEE Industrial Electronics Society Technical Committee on Industrial Agents, and his research interests are in the field of intelligent and reconfigurable production systems, cyber-physical systems and agent-based systems. 